Mini Review

\title{
TIBO derivatives as an anti HIV agent- QSAR and its utility
}

\begin{abstract}
TIBO derivatives played vital role in the therapy of HIV-1. This mini review deals with the earlier finding of QSAR. The article gone through many results and proposed various possibility of change in physiochemical, topological and 3D parameters in order to get better drug receptor interaction, inhibitory concentration and effective concentration. The findings support the existence of hydrophobic molecules, presence of halogen atom at $\mathrm{X}$ position with Balaben index.
\end{abstract}

Keywords: TIBO, Anti HIV, QSAR, topological parameter, inhibitory concentration, molecular modeling
Volume 7 Issue I - 2018

Lokendra Kumar Ojha

Regional Institute of Education, India

Correspondence: Lokendra Kumar Ojha, Regional Institute of Education, NCERT, Bhopal (MP), India, Email ojha.lokendra@gmail.com

Received: January 05, 2018| Published: February 08, 2018

\section{Introduction}

SAR stands for Structure Activity Relationship while the QSAR stands for Quantitative Structure Activity Relationship. SAR deals with the relationship of structure with biological activity while the QSAR accounts the relationship of magnitude of the various structural properties with the biological activity. Compounds with similar structures to a pharmacologically active drug are often themselves biologically active. This activity may be either similar to that of the original compound but different in potency and unwanted side effects or completely different to that exhibited by the original compound.

Drug discovery and development are expensive undertakings. The application of computational technology during drug discovery and development offers considerable potential for reducing the number of experimental studies required for compound selection and development and for improving the success rate. The quantitative structure activity relationships (QSAR) are certainly a major factor in contemporary drug design. Thus, it is quite clear why a large number of users of QSAR ${ }^{1,2}$ are located in industrial research units. So, Classical QSAR and 3D-QSAR are highly active areas of research in drug design..$^{3,4}$ In structure based drug design, one of the central strategies is to modify lead molecules slightly to obtain or improve certain therapeutic properties. ${ }^{5,6}$

\section{Utility of indicator parameter}

Indicator parameters are user defined parameters and used for presence or absence of certain group. The data were collected from the review article. ${ }^{7-9}$

$\log (1 / \mathrm{C})=1.4627( \pm 0.1473) \quad$ I_S $+1.2254 \quad( \pm$ $0.1443) \quad$ I_DMA $+0.7485 \quad( \pm 0.1798) \quad$ I_Cl+ 4.3417 $\mathrm{N}=74 \mathrm{R}=0.9170 \mathrm{Se}=0.5582 \mathrm{~F}=122.383$

Presence of sulfur atom (-S) leads to better activity than oxygen at five member ring, because of the higher electro negativity of the sulfur atom. Another important aspect of the model is that the -DMA substitution in place of -2MA, on seven member ring is definitely enhance the binding affinity of the drug. Presence of $-\mathrm{Cl}$ atom on benzene ring of the TIBO derivatives is really important to drug receptor binding affinity. So, all the three indicator parameter out of six, provide structural evidence for the modeling for the set of compound in present study.

\section{Utility of topological parameter}

Experimental anti-HIV activity (pIC50) complied from references. ${ }^{10-13}$

\begin{tabular}{|c|c|c|c|}
\hline & $( \pm .2$ & IX & \\
\hline & -3.0153 & $( \pm 2.8798)$ JhetZ & -3.2552 \\
\hline $13 \mathrm{R}=0.92$ & $0.4096 \mathrm{~F}=1$ & & Eq. (2) \\
\hline
\end{tabular}

From the result of the QSAR study, it appears that the presence of halogen atom at $\mathrm{X}$ position with Balaben index will increase the HIV1 binding affinity of TIBO derivatives. The most relevant structural conclusions of this study are the following: 1) presence of halogen atom on benzene ring of TIBO reduces the concentration; 2) Balaben type index (Jhetp and JhetZ) is essential to reduce the concentration.

\section{Utility of non-conventional topological parameter}

Experimental anti-HIV activity (pIC50) complied from references. ${ }^{10,11}$

$$
\begin{array}{cccccc}
\mathrm{pIC} 50= & 1.6601( \pm .5355) & \mathrm{IX} & + & 22.6684( \pm \\
12.3841) & \mathrm{RBF}+3.7816 & ( \pm & 2.9967) & \text { Yindex } & + \\
\mathrm{N}=16, \mathrm{r}=.7280, \mathrm{Se}=.9815, \mathrm{~F}=4.511 & & \multicolumn{2}{c}{\text { Eq. }(3)}
\end{array}
$$

Importance of indicator parameter (IX $=$ Presence of halogen at $\mathrm{X}$ position) is very important to enhance the biological activity of the drug. The halogen compounds are electro withdrawing in nature. Rotatable Bond Friction (RBF) is also very important phenomenon in order to get drug receptor binding affinity in particular sets of compound. The resulted QSAR model is best fit in particular set of compound and show the greater relationship with the inhibitory concentration (pIC50) and we can say that the presence of indicator parameter along with the RBF and Yindex is important non-conventional topological parameter and shows higher relationship.

\section{Utility of surface area and hydrophobicity}

16 derivatives of TIBO used in the present study. Experimental anti-HIV activity (pIC50) complied from references. ${ }^{10-12}$

$$
\begin{array}{cccr}
\mathrm{pIC} 50= & -2.7766( \pm .3910) & \mathrm{MnPR} & + \\
7.9132( \pm .1 .4746) & \mathrm{J} 3 \mathrm{D} \quad-.0255( \pm .0096) & \mathrm{ASA}+ & + \\
\mathrm{N}=15, \mathrm{R}=.9421, \mathrm{Se}=.4981, \mathrm{~F}=28.945 & \multicolumn{2}{c}{\text { Eq. (4) }}
\end{array}
$$

For the dataset of 16 analogues with well-defined activity, the HIV1 non nucleoside reverse transcriptase inhibitory potency appears to 
be influenced by structural components. The interpretation of the generated QSAR revealed that increased HIV-1 inhibitory potency of TIBO derivatives could be achieved by increasing solvent accessible surface area of the molecules and by Minimal Projection Radius $\AA 2$ in the molecule.

$\begin{array}{cccr}\text { IC } 50= & 1.5734( \pm .1930) & \text { IS } & +.6355 \\ ( \pm .1176) & \operatorname{logP} \quad+.0082( \pm .0023) & \text { SAG-1.2184 } \\ \mathrm{N}=89, \mathrm{R}=.8328, \mathrm{SEE}=.8086, \mathrm{~F}=64.104 & & \text { Eq. }(5)\end{array}$

The findings support the existence of hydrophobic molecules in this series would have been advantageous for increasing the activity. The X-substituents at ring A have wide variation and are at different positions. All of them are shown to effect the activity by their hydrophobic property, but those at the 8-position are shown to also have some additional positive steric effect. $\log \mathrm{P}$ is a measure of hydrophobicity which is important for the penetration and distribution of the drug, but also for the interaction of drug with receptors. Quantitative structure activity relationship study of anticonvulsant activity of $\alpha \_$substituted acetamido-N-benzylacetamide derivatives are also shows great results in this concern. ${ }^{14}$

\section{Conclusion}

All finding of various sets of TIBO derivatives are in support of utility of indicator, topological, non-conventional topological and surface area properties. This results proposed a good QSAR model and their synthesis can be done by keeping view of these parameters. All though the final idea about the synthesis of compound may come only after the complete study viz 3D QSAR, molecular docking and other computational techniques. These guidelines may therefore, provide a basis for rationalizing substituent selection in the future designing of selective TIBO receptor ligands. The study may also help in proposing the possible mode of action of TIBO.

\section{Acknowledgments}

None.

\section{Conflicts of interest}

The authors declare no conflicts of interest.

\section{References}

1. Trinajstic N. Chemical Graph Theory. 1992. pp. 225-273.
2. Bazoui H, Zahouily M, Boulaajaj S, et al. QSAR for anti-HIV activity of HEPT derivatives. SAR and QSAR in Environmental Research. 2002;13(6):567-577.

3. Kubinyi H. QSAR: Hansch analysis and related approaches. In: Mannhold R, et al. editors. Methods and principles in medicinal chemistry. Wiley, Weinheim, Germany. 1993

4. Kubinyi H. 3D QSAR in drug design: theory, methods and applications. ESCOM, Leiden, Springer publisher, Netherlands. 1993.

5. Klebe G. Virtual ligand screening:strategies, perspectives and limitations. Drug Discov Today. 2006;11(13-14):580-594.

6. Jorgensen WL Efficient drug lead discovery and optimization. Accounts of Chemical Research. 2009;42(6):724-733.

7. Kukla MJ, Breslin HJ, Pauwels R, et al. Synthesis and anti-HIV-1 activity of 4,5,6,7-tetrahydro-5-methylimidazo[4,5,1-jk][1,4] benzodiazepin-2(1H)-one (TIBO) derivatives. Journal of medicinal Chemistry. 1991;34(2):746-751.

8. Kukla MJ, Breslin HJ, Pauwels R, et al. Synthesis and anti-HIV-1 activity of 4,5,6,7-tetrahydro-5-methylimidazo[4,5,1-jk][1,4] benzodiazepin-2(1H)-one (TIBO) derivatives. Journal of medicinal Chemistry. 1991;34(11):3187-3197.

9. Breslin HJ, Kukla MJ, Ludovici DW, et al. Synthesis and anti-HIV1 activity of 4,5,6,7-tetrahydro-5-methylimidazo[4,5,1-jk][1,4] benzodiazepin-2(1H)-one (TIBO) derivatives. Journal of medicinal. 1995;38(5):771-793.

10. Mahmoudian M. The cannabinoid receptor:Computer-aided molecular modeling and docking of ligand. Journal of Molecular Graphics and Modelling. 1997;15(3):149-153.

11. Zhou Z, Madrid M, Madura JD. Docking of non-nucleoside inhibitors:neotripterifordin and its derivatives to HIV-1 reverse transcriptase. Proteins. 2002;49(4):529-542.

12. Smith MBK, Lamb ML, Tirado-Rives J, et al. Monte Carlo calculations on HIV-1 reverse transcriptase complexed with the non-nucleoside inhibitor 8-Cl TIBO:contribution of the L100I and Y181C variants to protein stability and biological activity. Peds. 2000;13(6):413-421.

13. Titmuss SJ, Keller PA, Griffith R. Docking experiments in the flexible non-nucleoside inhibitor binding pocket of HIV-1 reverse transcriptase. Bioorganic \& Medicinal Chemistry. 1999;7(6):1163-1170.

14. Abdulfatai U, Uzairu A, Uba S. Quantitative structure activity relationship study of anticonvulsant activity of $\alpha$ substituted acetamido-N-benzylacetamide derivatives. Cogent Chemistry. 2013. 